\title{
Relation entre l'état de réplétion digestive et la composition hépatique du canard mulard en gavage
}

\author{
Alain Auvergne*, Michel Candau, René Babile, Hélène Manse, \\ Michel Bouillier-Oudot
}

\begin{abstract}
Laboratoire de zootechnie et qualité des produits animaux, Ensa de Toulouse, 145, avenue de Muret, 31076 Toulouse cedex 3, France
\end{abstract}

(Reçu le 22 mai 1997 ; accepté le 20 janvier 1998)

\begin{abstract}
Relationship between crop repletion and hepatic composition in force-fed mule ducks. Two batches of male mule ducks were constituted on the basis of the filling state of the crop at slaughter, after being over-fed twice a day for 5 days: no corn in the crop defined ' $O V$ ' birds, and corn in the crop defined 'OP' birds. 'OP' animals presented significantly lower daily consumption during the last 3 days of force-feeding. They had a lighter liver weight than ' $O V$ ' ducks (178 versus $197 \mathrm{~g}$ ), lower hepatic lipid percentages ( 25.0 versus $33.0 \%$ ), and enhanced hepatic glycogen rates $(4.7$ versus $2.4 \%$ ). Metabolic state of animals might explain these variations in hepatic composition. Unadaptation to force-feeding modifies the metabolic inbalance of the overfed animals and consequently induces higher levels of carbohydrate. These results suggest that similar differences between crude fatty livers could be reflected in the quality of the cooked products. (C) Inra/Elsevier, Paris
\end{abstract}

glycogen / glucose / liver / duck / over-feeding / lipids / digestion

Résumé - Vingt canards mulards mâles sont gavés pendant $5 \mathrm{j}$ puis abattus $12 \mathrm{~h}$ après le dixième repas. À l'abattage, deux lots sont constitués sur la base de l'état de réplétion de l'oesophage terminal : les animaux sans maïs dans l'œesophage «OV » et les animaux ayant du maïs, «OP ». Les animaux « $\mathrm{OP}$ » présentent des consommations quotidiennes significativement plus faibles lors des trois derniers jours de gavage, ont un poids de foie inférieur à celui des animaux « $\mathrm{OV}$ » (178 vs $197 \mathrm{~g}$ ), des teneurs en lipides hépatiques plus faibles ( 25,0 vs $33,0 \%$ ), des teneurs en glycogène hépatique plus élevées $(4,7$ vs $2,4 \%$ ). Ces différences traduisent une inadaptation à la suralimentation forcée des canards « $\mathrm{OP}$ » et une modification de l'équilibre métabolique de l'ani-

\footnotetext{
* Correspondance et tirés à part.

E-mail: auvergne@flora.ensat.fr
} 
mal. Cela conduit à des différences précoces de composition hépatique (notamment en glucides) qui pourraient expliquer en partie l'homogénéité très variable des lots de foies gras en production. (C) Inra/Elsevier, Paris

\section{glycogène / glucose / foie / canard / sur alimentation / lipides / digestion}

\section{INTRODUCTION}

De nombreuses études ont mis en évidence le bouleversement métabolique provoqué par le gavage des palmipèdes gras $[5,6,10]$. L'incidence de certains facteurs sur le développement de la stéatose [3], et du développement corporel des canards gavés [1] ont été mis en évidence.

Ainsi la réponse des animaux à la suralimentation forcée est variable les premiers jours du fait d'une difficulté d'adaptation de certains animaux. Cela se traduit par des écarts de consommation qui expliquent en partie le développement de la stéatose et par suite la productivité pondérale [4]. Cette capacité d'adaptation peut être appréciée par l'état de réplétion de l'csophage distal $12 \mathrm{~h}$ après le dernier repas, sachant que la vidange complète de cet organe est pratiquement effectuée en $24 \mathrm{~h}$ che $\mathrm{z}$ le canard de Barbarie et en $12 \mathrm{~h}$ chez le mulard dont le transit digestif est plus rapide [2].

L'objet de cette étude est d'apprécier l'incidence de l'état de réplétion du tube digestif sur certains paramètres zootechniques et sur la composition hépatique, chez des canards mulards après $5 \mathrm{j}$ de suralimentation forcée.

\section{MATÉRIEL ET MÉTHODES}

\subsection{Conduite expérimentale des animaux}

L'expérimentation est effectuée avec des canards mulards mâles issus d'un croisement entre un mâle Barbarie (souche R31) et une cane Pékin (souche Alienor). Les animaux sont pesés le jour de la mise en gavage après un jeûne de 18 h (PMEG).

\subsubsection{Gavage}

Le gavage est effectué en cage individuelle dans un bâtiment de type traditionnel recevant une ventilation dynamique $\left(50 \mathrm{~m}^{3} / \mathrm{h} /\right.$ canard les 3 premiers jours, puis $120 \mathrm{~m}^{3} / \mathrm{h} /$ canard). Les animaux sont gavés pendant $5 \mathrm{j}$ (dix repas) avec du maïs cuit au moyen d'une gaveuse électromécanique de type "israélien ». Les animaux ne reçoivent aucune médication à but prophylactique avant et pendant le gavage. L'enregistrement des consommations individuelles en gavage est effectué au moyen d'un automate de pesée relié à la gaveuse.

\subsubsection{Abattage et constitution des groupes}

L'abattage des animaux est réalisé $12 \mathrm{~h}$ après le $10^{\mathrm{e}}$ repas. Après pesée (PVabattage), les animaux, étourdis par électronarcose, sont saignés par section des carotides et des jugulaires à la base de la tête. Ils sont ensuite trempés dans un bac d'eau chaude et plumés à l'aide d'une plumeuse électrique. Les animaux sont éviscérés entre 15 et 30 min après la mort. Ils sont ensuite disséqués selon la méthode décrite par Ricard [14], ce qui permet de mesurer les poids de foie, de la masse viscérale (intestins et mésentère), du gras pariétal (gras abdominal), du pectoral superficiel (peau + gras sous-cutané et muscle), de l'ensemble cuisse-pilon.

Deux groupes de huit animaux sont constitués selon l'état de réplétion de l'cesophage : les animaux dits « $O P$ » qui sont des animaux dont le tube digestif antérieur contient du maïs et qui n'ont donc pas terminé leur digestion ; les animaux dits « $\mathrm{OV} »$ qui sont des animaux sans maïs dans ce compartiment. Le maïs présent dans l'œsophage distal et le ventricule succenturié est pesé. À partir de ces enregistrements, les paramètres suivants ont été calculés : 
- la consommation totale et la consommation/PMEG $(\mathrm{kg} / \mathrm{kg})$,

- la quantité de maïs présente dans le tube digestif antérieur,

- le gain de poids corrigé par la quantité de maiis présente dans le tube digestif antérieur (GPnet).

Après pesée du foie, un échantillon de $1 \mathrm{~cm}$ d'épaisseur est prélevé dans la partie médiane du grand lobe selon un axe longitudinal. Il est immédiatement congelé dans l'azote liquide, puis broyé et conservé à $-52{ }^{\circ} \mathrm{C}$ jusqu'à l'analyse.

\subsubsection{Paramètres de la composition hépatique}

Des analyses biochimiques concernant la composition hépatique sont réalisées sur les différents prélèvements de chaque foie.

Sur l'échantillon entier, on détermine les taux de matière sèche, matières azotées totales (MAT) et de lipides totaux.

- Matière sèche : la détermination de la matière sèche est effectuée par dessiccation à l'étuve à $103^{\circ} \mathrm{C}$ pendant $12 \mathrm{~h}$ (Afnor NF 04-401-1968).

- Matières azotées totales $(\mathrm{N} \times 6,25)$ : après minéralisation selon la méthode de Kjeldhal, le sulfate d'ammonium formé est dosé par colorimétrie $(660 \mathrm{~nm})$ selon Verdouw et al. [15].

- Les lipides totaux : le dosage est réalisé selon la méthode de Folch et al. [9].

Le fractionnement des éléments structuraux et cellulaires du tissu hépatique est effectué selon la méthode décrite par Lewis et al. [11] adaptée aux caractéristiques des foies stéatosés [13]. Après séparation du contenu lipidique des hépatocytes (par formation d'une couche de graisse semi-solide, ou fat-cake), deux phases sont obtenues après homogénéisation et centrifugation (15 min-2 $300 \mathrm{~g})$ du sous-nageant : une phase dite $\mathrm{S} 1$ contenant des éléments cellulaires solubles et un culot $\mathrm{C} 1$ contenant les structures membranaires de l'hépatocyte.

- Les matières azotées totales du surnageant $\mathrm{S} 1$ et du culot membranaire $\mathrm{C} 1$ sont estimées selon la méthode sus-décrite.

- Les protéines présentes dans le surnageant S1 sont estimées par la méthode de Lowry et al. [12] avec le kit Protein assay (Sigma Diagnostics P5656).
- Les taux de glucides totaux (glycogène, glucose et glucose-6-phosphate) sont déterminés dans la fraction du surnageant $S 1$ selon la méthode décrite par Dalrymple et Hamm [7] : le glycogène, après hydrolyse en $n$ glucose par l'amyloglucosidase (Aspergillus niger - Boehringer 208-469) ainsi que le glucose et le glucose-6-phosphate, contribuent, en présence d'hexokinase et de glucose-6-phosphate déshydrogénase, à la production de gluconate-6-phosphate et de coenzyme réduit NADPH. Ce métabolite est dosé par spectrophotométrie à $340 \mathrm{~nm}$ (kit glucose Boehringer 716.251). Le taux de glucose libre (glucose et glucose-6-phosphate) est dosé séparément. Le taux de glycogène est ensuite estimé par différence entre les concentrations de glucose total et de glucose libre (exprimées en $\mu \mathrm{mol}$ d'équivalent glucose).

\subsection{Analyses statistiques}

L'incidence de l'état digestif des animaux lors de l'abattage est étudiée par analyse de variance selon la procédure du modèle linéaire généralisée (logiciel Minitab).

\section{RÉSULTATS}

\subsection{Caractéristiques zootechniques et composition de la carcasse}

Les données correspondant aux paramètres zootechniques et à la composition de la carcasse sont reportées sur le tableau I et la figure 1. Les animaux ont un poids vif moyen à la mise en gavage (PMEG) inférieur au potentiel de ce type génétique à cet âge $(4,2 \mathrm{~kg}$ en moyenne).

Les canards dits « $\mathrm{OV} »$ présentent une consommation quotidienne ou cumulée supérieure (figure 1). La vacuité de l'œsophage témoigne de la digestion presque totale de la ration. À l'opposé, les canards présentant beaucoup de maïs dans le tube digestif antérieur (animaux dits « OP ») ont eu des consommations globalement plus faibles. Le critère consommation 
Tableau I. Paramètres zootechniques et de découpe en fonction de la réplétion de l'œsophage (huit canards par lot).

\begin{tabular}{|c|c|c|c|c|}
\hline & $\mathrm{OP}$ & OV & Sres & Stat* \\
\hline \multicolumn{5}{|l|}{ Paramètres zootechniques } \\
\hline PMEG $(k g)$ & 3,80 & 3,82 & 0,16 & ns \\
\hline PVabattage $(\mathrm{kg})$ & 5,04 & 4,68 & 0,23 & $* *$ \\
\hline GPnet $(\mathrm{kg})$ & 0,76 & 0,81 & 0,00 & ns \\
\hline Maïs dans le tube digestif antérieur (kg) & 0,49 & 0,06 & 0,12 & $* *$ \\
\hline Consommation totale $(\mathrm{kg})$ & 3,40 & 3,84 & 0,07 & $* *$ \\
\hline Consommation/PMEG (kg/kg) & 0,90 & 1,01 & 0,05 & $* *$ \\
\hline \multicolumn{5}{|l|}{ Paramètres de découpe } \\
\hline Intestin + mésentère (g) & 317 & 288 & 33 & $* *$ \\
\hline Gras abdominal $(\mathrm{g})$ & 116 & 113 & 21 & ns \\
\hline Foie $(g)$ & 178 & 197 & 20 & $* *$ \\
\hline Peau du pectoral $(\mathrm{g})$ & 92 & 94 & 12 & ns \\
\hline Muscle du pectoral (g) & 224 & 226 & 16 & ns \\
\hline Cuisse-pilon (g) & 334 & 350 & 24 & $\mathrm{~ns}$ \\
\hline
\end{tabular}

* Signification statistique : ${ }^{* *} p=<0,05 ; \mathrm{ns}=$ non significatif.

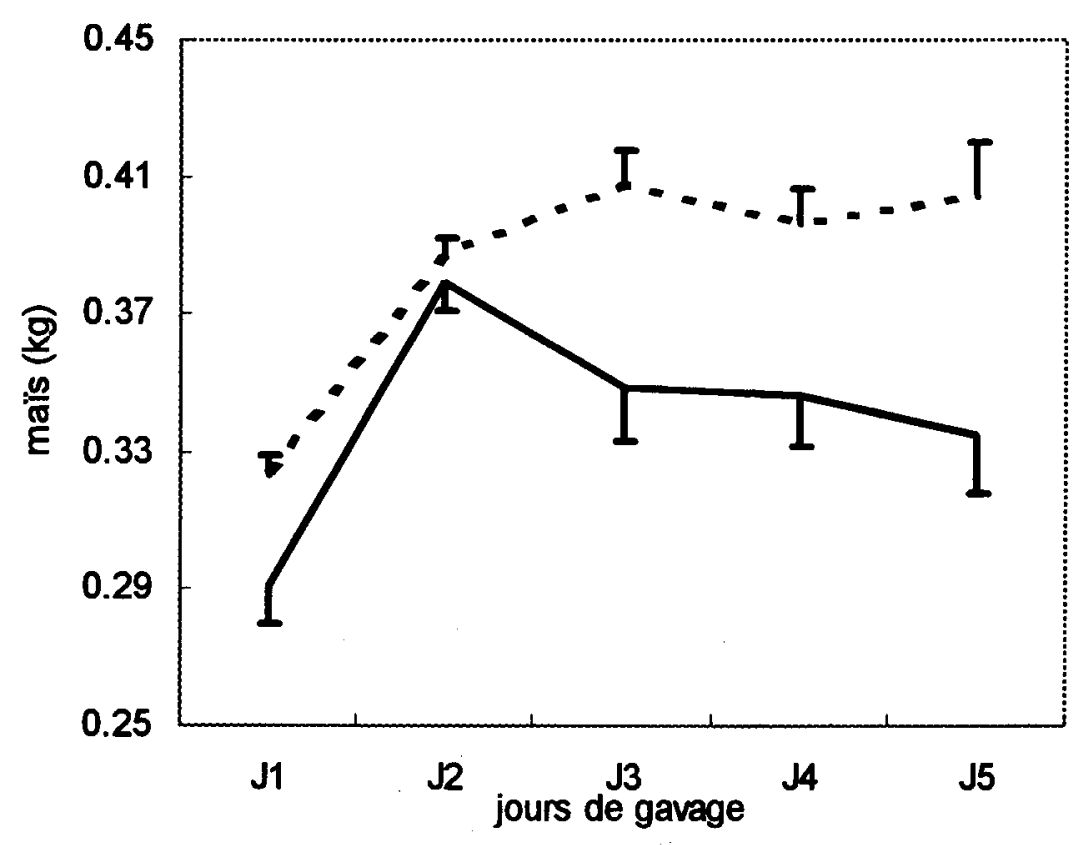

Figure 1. Repas moyen quotidien des animaux (moyenne \pm écart type de la moyenne, en $\mathrm{kg}$ ). animaux « OP », - - - - animaux « OV». 
cumulée discrimine parfaitement les deux types d'animaux (figure 2).

Dans le groupe des animaux « $\mathrm{OV}$ » l'ingestion est environ de $1,01 \mathrm{~kg}$ de maïs/kg de PMEG en $5 \mathrm{j}$ alors que, dans l'autre lot, elle n'est que de $0,90 \mathrm{~kg} / \mathrm{kg}$ PMEG $(p=0,01)$. Les consommations quotidiennes ne sont cependant significativement différentes que lors des 3 derniers jours entre les deux lots (figure l).

Les canards « $\mathrm{OP} »$ ont un poids vif à l'abattage très supérieur à celui des animaux « OV » $(5,04$ vs $4,68 \mathrm{~kg}$, tableau $)$. Cependant, en raison de la présence de quantité importante de maiis dans le tube digestif antérieur $(0,49 \mathrm{vs} 0,06 \mathrm{~kg})$, les gains de poids nets (GPnet) ne sont pas statistiquement différents entre les deux lots : $(0,76$ vs $0,81 \mathrm{~kg})$.
Au niveau des paramètres de découpe, seuls le poids de l'ensemble intestin + mésentère et le poids de foie sont significativement différents entre les deux lots : les « OP » présentant un poids de tube digestif supérieur et un poids de foie inférieur par rapport aux animaux « $\mathrm{OV} »$.

\subsection{Composition hépatique}

\subsubsection{Teneurs en eau et lipides totaux}

Les animaux « $\mathrm{OV}$ » ont un poids de foie supérieur à celui des animaux « $\mathrm{OP}$ » (197 vs $178 \mathrm{~g}$, tableau II). Les différences de poids de foie se retrouvent au niveau de la teneur en lipides totaux. Les animaux «OP» présentent $27 \%$ de lipides de moins que les animaux «OV ». Corréla-

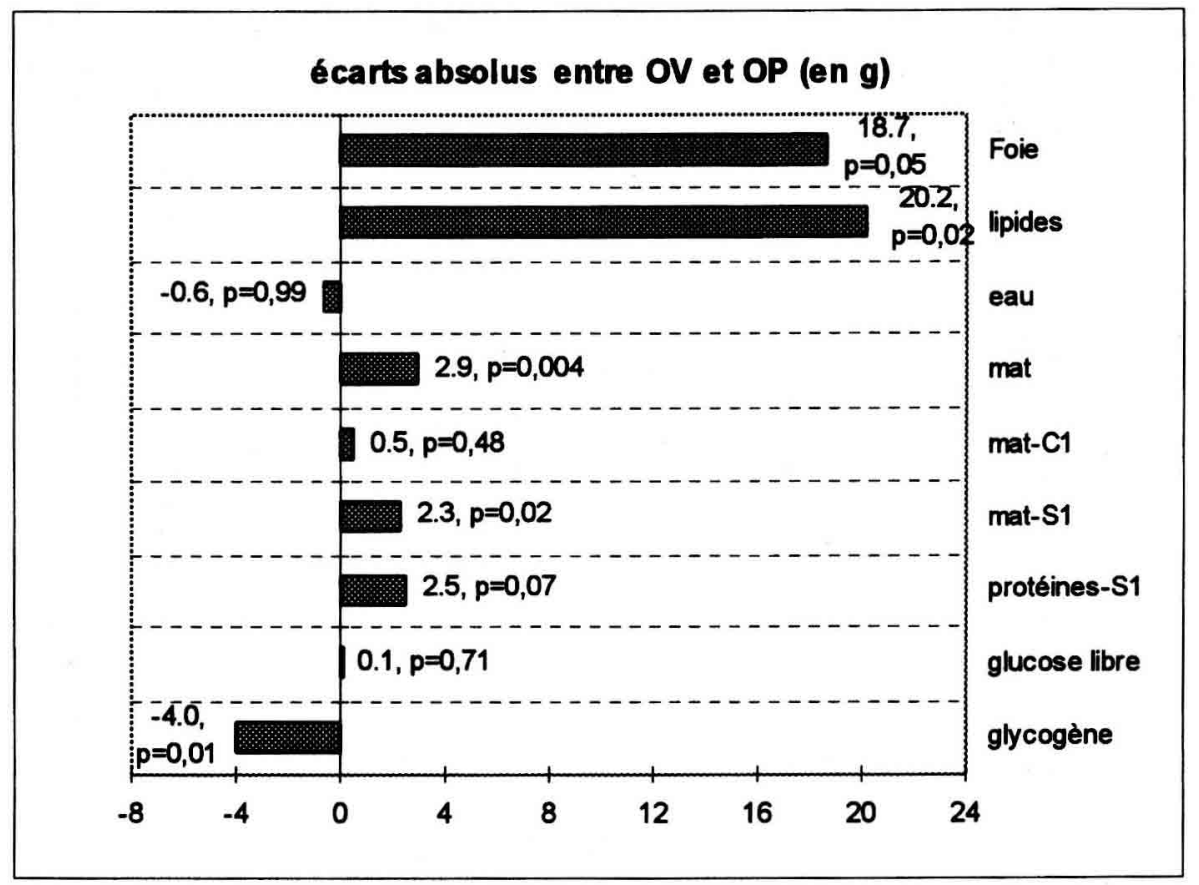

Figure 2. Effet de l'état de réplétion digestive sur les quantités de constituants hépatiques (OV$\mathrm{OP}$, écart en g et probabilité Ho associée). 
Tableau II. Composition hépatique des canards en fonction de la réplétion de l'œsophage (huit canards par lot).

\begin{tabular}{lcccc}
\hline & OP & OV & Sres & Stat* \\
\hline Poids foie (g) & 178 & 197 & 20 & $* *$ \\
Composition brute en $\mathrm{mg} / \mathrm{g}$ de foie & & & & \\
$\quad$ Lipides & 250 & 330 & 52 & $* *$ \\
$\quad$ Humidité & 567 & 502 & 32 & $* *$ \\
MAT ech & 107 & 112 & 9 & $\mathrm{~ns}$ \\
MAT C1 & 32 & 32 & 10 & $\mathrm{~ns}$ \\
MAT S1 & 77 & 81 & 11 & $\mathrm{~ns}$ \\
Protéines S1 & 75 & 81 & 10 & $\mathrm{~ns}$ \\
Glucose libre & 14 & 13 & 2 & $\mathrm{~ns}$ \\
Glycogène & 47 & 24 & 14 & $* *$ \\
Composition du foie délipidé en mg/g & & & & \\
Humidité & 760 & 812 & 4 & $* *$ \\
MAT & 140 & 168 & 19 & $* *$ \\
MAT Cl & 42 & 48 & 14 & $\mathrm{~ns}$ \\
MAT S1 & 101 & 120 & 11 & $* *$ \\
Protéines S1 & 98 & 121 & 16 & $* *$ \\
Glucose libre & 18 & 19 & 2 & $\mathrm{~ns}$ \\
Glycogène & 61 & 36 & 19 & $* *$ \\
\hline
\end{tabular}

MAT : Matières azotées totales du tissu. MAT C1 teneur en MAT du culot membranaire. MAT S1 : teneur en MAT du surnageant. Protéines S1 : teneur en protéines du surnageant. * Signification statistique ; ${ }^{* *} p<0,05 ; \mathrm{ns}$ : non significatif.

tivement, la teneur en eau est supérieure de $7 \%$ chez les animaux « OP ». Cependant, la teneur en eau du foie délipidé est significativement plus importante chez les animaux « $\mathrm{OV} »(812$ vs $760 \mathrm{mg} / \mathrm{g}$ de foie, $p<0,05$ ).

\subsubsection{Teneur en azote}

Globalement, il n'y a aucune différence significative de teneur en matières azotées totales ou en protéines selon les fractions tissulaires et selon l'état de digestion des animaux.

Cependant, dans la fraction délipidée, les taux en matières azotées totales du tissu sont supérieurs chez les animaux « $\mathrm{OV}$ » (168 vs $140 \mathrm{mg} / \mathrm{g}$ ). Cette différence pro- vient principalement de la teneur en matières azotées totales du surnageant $S 1$ (MAT-S1 = 120 vs $101 \mathrm{mg} / \mathrm{g}$ ) alors que l'on n'observe pas d'écart significatif au niveau du culot membranaire $\mathrm{C} 1$ (42 vs $48 \mathrm{mg} / \mathrm{g}$ foie délipidé). La même différence est observée dans le cas de la teneur en protéines ( $121 \mathrm{vs} 98 \mathrm{mg} / \mathrm{g}$ ). Le calcul des quantités de constituants hépatiques confirme ces résultats (figure 2).

\subsubsection{Teneurs en glycogène et glucose libre}

Aux deux états digestifs correspondent des taux de glycogène significativement différents quel que soit le mode d'expression des résultats, en composition brute 
du foie ou en fonction du poids de foie délipidé. Les animaux « $O P$ » présentent des teneurs en glycogène supérieures à celles des animaux «OV », $+48 \%$ et $+42 \%$ respectivement en brut et en poids de foie délipidé. En revanche, il n'y a pas de différence pour les taux de glucose libre (glucose + glucose-6-phosphate) quel que soit le mode d'expression : tissu frais ou tissu délipidé.

\section{DISCUSSION}

\subsection{Relation entre l'état de réplétion du tube digestif antérieur et les paramètres zootechniques}

Pendant les trois premiers jours de gavage, le gaveur a augmenté fortement les quantités distribuées, entraînant des adaptations digestives différentes selon les canards. Certains supportent le rythme alimentaire imposé et, après 10 repas, sont capables d'évacuer leur ration en moins de $12 \mathrm{~h}$ (animaux « OV »), et ne contiennent pas d'aliment dans l'œsophage lors du repas suivant (le délai de jeûne correspondant à l'intervalle entre deux repas).

À l'opposé, un certain nombre d'animaux est en situation d'embarras gastroœsophagien, caractérisée par la présence d'une fraction importante de la ration d'un repas à l'autre. On retrouve dans le tube digestif antérieur $489 \mathrm{~g}$ de maïs correspondant au dernier repas de $405 \mathrm{~g}$. Cet état se traduit par une réplétion légèrement plus importante de la masse intestinale $(+29 \mathrm{~g} ; p<0,05)$. On peut penser que cet écart n'est pas lié à un engraissement mésentèrique supérieur dans la mesure où la masse du gras abdominal est identique (116 et $113 \mathrm{~g}$ ) dans les deux groupes. A contrario, les poids de foies sont légèrement inférieurs dans ce groupe $(-19 \mathrm{~g} ; p<0,05)$.
Ces écarts semblent donc correspondre à des potentialités d'adaptation digestive différentes qui pourraient être associées à différents niveaux d'absorption intestinale et consécutivement de lipogénèse hépatique [2]. La consommation cumulée peut donc rendre compte du développement du foie gras, plus important chez les canards à osophage vide.

\subsection{Relation entre l'état de réplétion digestive et la composition hépatique}

On note une accumulation plus importante de lipides hépatiques chez les animaux « $O V$ » $(33,0$ vs $25,0 \%)$ et un taux de glycogène plus faible $(2,4$ vs $4,7 \%)$. Le rythme d'assimilation de l'aliment semble donc modifier le métabolisme hépatique. Pour un faible rythme d'assimilation, le foie synthétise des lipides qu'il peut exporter facilement, ce qui expliquerait que les animaux «OP » aient des teneurs en lipides inférieures. Nos résultats (figure 3) montrent nettement que la teneur en lipides augmente, alors que celle en glycogène évolue en sens inverse $\left(r^{2}=-0,70, p<0,001\right)$. En d'autres termes, la glycogénogenèse hépatique est progressivement remplacée par la lipogenèse, lorsque la pression alimentaire est plus importante. Cela corrobore les observations faites par Durand [8]. Les animaux «OV » seraient donc dans une phase de synthèse lipidique. Les consommations alimentaires des animaux « $\mathrm{OP}$ », nettement plus faibles lors des trois derniers jours pourraient expliquer la moindre accrétion lipidique, la vitesse de progression des ingérés étant déterminante au niveau de la stéatose hépatique des animaux gavés [1].

Les quantités de matières azotées totales du foie des animaux « $\mathrm{OV}$ » sont supérieures à celles des animaux « OP » (figure 2). Cette différence est attribuable 


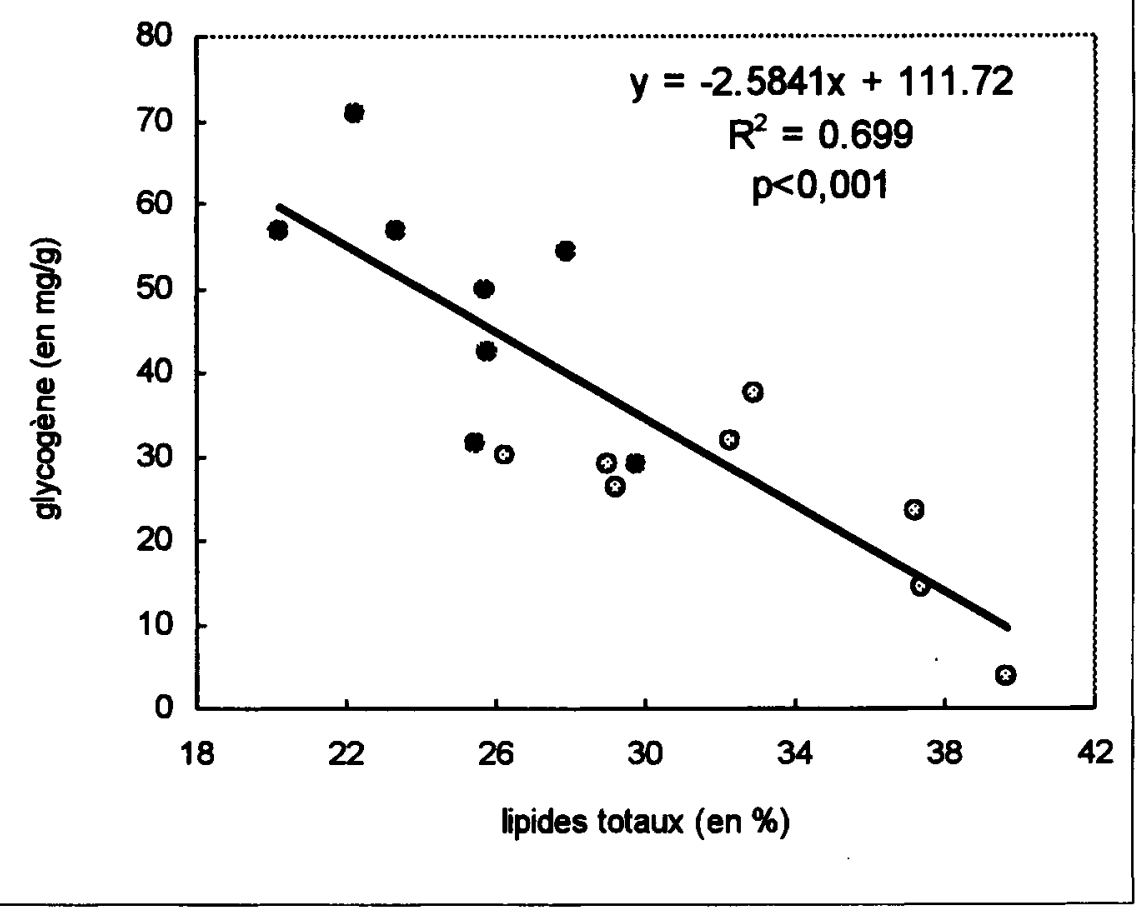

Figure 3. Évolution relative des taux de lipides totaux et de glycogène hépatiques.

aux matières azotées totales du contenu cellulaire (MAT S1) qui pour les animaux «OV » sont presque constituées exclusivement de protéines $(121 \mathrm{mg} / \mathrm{g}$ foie délipidé comparé à $120 \mathrm{mg} / \mathrm{g}$ ). On peut penser que cela traduit des activités de synthèse plus importantes chez les animaux « $\mathrm{OV}$ » au niveau protéique comme en ce qui concerne la lipogenèse. Les effets de l'état de réplétion digestive ne sont pas décelables sur les matières azotées de structure (MAT C1) soit parce que le gavage n'est pas assez avancé, soit parce que les animaux ont des poids à la mise en gavage inférieurs aux valeurs usuelles pour ce type d'animaux. Dans ce cas, la croissance azotée musculaire reste prioritaire sur celle du foie [1]. Le fait qu'au niveau des para- mètres de découpe le poids des muscles pectoraux contrairement à celui du foie ne soit pas différent entre les deux groupes de canards appuie cette hypothèse.

En conclusion, l'influence de l'état de réplétion du tube digestif $12 \mathrm{~h}$ après le repas est surtout marquée sur le poids de foie ainsi que sur la composition hépatique. Une inadaptation à la suralimentation forcée conduit à un œsophage terminal encombré et des poids de foie moindres, associés à des taux de lipides hépatiques plus faibles et de glycogène plus élevés. Dans la mesure où ces écarts perdureraient jusqu'à la fin du gavage, ceci pourrait expliquer en partie l'hétérogénéité des lots de foie gras en production. 
Ce travail souligne donc l'importance de la vidange gastro-œsophagienne, et sa relation avec l'assimilation digestive, chez le canard en gavage : les différences sontelles anatomiques ou fonctionnelles, leurs conséquences sont-elles récessives ou définitives ? Une étude de la motricité du tube digestif antérieur et de ses facteurs de variation permettrait de préciser à quel niveau se situe le frein limitant le transit en comparant des canards « $\mathrm{OP} »$ et $« \mathrm{OV} »$.

\section{REFERENCES}

[1] Auvergne A., Facteurs de variation de la composition corporelle et tissulaire de canards avant et après gavage, thèse de doctorat d'État, INP Toulouse, 1992, 252 p.

[2] Auvergne A., Babilé R., Remond D., Latil G., Digestibilité du maiss en gavage. Eléments de prédiction des gains de poids, C.R. Journées de la Recherche sur les Palmipèdes Gras, Bordeaux, avril 1993, Inra-Itavi-Cifog, volume $1,77-89$.

[3] Babilé R., La production de foies gras de canards de Barbarie (Cairina moschata). Aspects génétiques, nutritionnels et technologiques, thèse de doctorat d'État, INP Toulouse, 1989, $231 \mathrm{p}$.

[4] Babilé R., Auvergne A., Delpech P., Meirieu $O$., Evolution de la consommation de maïs au cours du gavage et incidence sur la production de foie gras chez le canard de Barbarie, Ann. Zootech. 36 (1) (1987) 73-84

[5] Blum J.C., Leclercq B., Nouvelles précisions sur les modifications biochimiques et histologiques du foie provoquées par le gavage, in : Journées Avicoles de Varèse, 31 mai-4 juin, 2, 1973, 193-207.
[6] Blum J.C., Leclercq B., Graf B., Study of the biochimical and histochemical process leading to the formation of a fatty liver in the force feed goose, in : Process of the 42nd Meeting of the Am. Oil Chemist. Soc., New York, October 1968, $n^{\circ} 73,1968,45$.

[7] Dalrymple R.H., Hamm R., A method for the extraction of glycogen and metabolites from a single muscle sample, J. Fd Technol. 8 (1973) 439-444.

[8] Durand P., Évolution du foie au cours du gavage - Session palmipèdes et foies gras, 8-9 mai, Brives, Itavi Ed., 1979, 3 p.

[9] Folch J., Lees M., Sloane-Stanley G.H.S., A simple method for the isolation and purification of the total lipids from animals tissues, J. Biol. Chem. 226 (1957) 498-509.

[10] Leclercq B., Durand C., Delpech P., Blum J.C., Notes préliminaires sur l'évolution des constituants biochimiques du foie au cours du gavage de l'oie, Ann. Biol. Anim. Bioch. Biophys. 8 (4) (1968) 549-556.

[11] Lewis D.S., Celluci M.D., Masoro E.J., Yu B.P., An improved method for the isolation of adipocyte plasma membranes, Anal. Biochem. 96 (1979) 236-245.

[12] Lowry O.H., Rosebrough N.J., Farr A.L., Randall R.J., Protein mesurement with the folin phenol reagent, J. Biol. Chem. 193 (1951) 265-275.

[13] Pech G., Contribution à l'étude des sucres du foie gras. Evolution post mortem en fonction de facteurs zootechnique et technologique, thèse de D.R.U., INP Toulouse, 1996, 62 p.

[14] Ricard F.H., Essai d'estimation directe des différents éléments de la carcasse de poulet en vue d'apprécier son rendement en viande, Ann. Zootech. 13 (4) (1964) 355-366.

[15] Verdouw H., Van Echteld C.J.A., Dekkers E.M.J., Ammonia determination based on indophenol formation with sodium salicylate, Water. Res. 12 (1977) 399-402. 